\title{
Using Period-Luminosity-Metallicity relations to Calculate Distance for the RR Lyrae Star, EZ Lyr
}

\author{
Kaitlyn E. KIDWELL ${ }^{1, *}$ And Mary F. KIDD ${ }^{1}$ \\ ${ }^{1}$ Tennessee Technological University, College of Art and Sciences, Department of Physics, Cookeville, Tennessee, USA \\ "Corresponding author: kekidwell42@students.tntech.edu
}

\begin{abstract}
Reliable methods to measure intergalactic distances are a valuable resource in the field of astronomy. Periodic variables stars, such as RR Lyraes, can be used as standard candles to determine the distance to the stellar structures where they are located. For this reason, many different student groups observed and analyzed RR Lyrae stars through a research course offered by Our Solar Siblings, and the focus of this particular study was the star EZ Lyr. Images of EZ Lyr were taken by Las Cumbres Observatory and processed using tools provided for the research course. Light curves were produced in B, V, sdss-i and sdss-z filters that were analyzed to determine the star's period and luminosity. The period-luminosity-metallicity relations described in Caceres and Catelan (2008) were used to calculate the distance to EZ Lyr in each filter. The final distance measurement, $1406 \pm 32 \mathrm{pc}$ was slightly closer $(\approx 100 \mathrm{pc})$ compared to results from the GAIA satellite.
\end{abstract}

(a)(1) $\odot 2021$ Astronomy Theory, Observations and Methods Journal

Keywords: $\quad$ stars - variables - RR Lyrae - photometric telescopes

https://doi.org/10.32374/atom.2020.2.5

\section{INTRODUCTION}

RR Lyrae stars are a class of pulsating variables often used as standard candles and can be useful in determining the distance to many stellar structures such as globular clusters within the galaxy as well as providing valuable information about their metallicities (Fitzgerald et al., 2012). They typically have a spectral type range from $\mathrm{A}$ to $\mathrm{F}$ and a pulsation period between 0.2 to 1.2 days (Dambis et al., 2013). (Cáceres \& Catelan, 2008) calculated a relation between period-luminosity and distance using the Sloan Digital Sky Survey (SDSS), particularly in the i and $\mathrm{z}$ filters. Limited data has been observed using this relation, making measurements using these relations relatively untested. The mathematical relations proposed by (Cáceres \& Catelan, 2008):

$$
\begin{gathered}
M_{i}=0.908-1.035 \log P+0.220 \log Z \\
M_{z}=0.839-1.95 \log P+0.211 \log Z \\
\log Z=[M / H]-1.765 \\
{[M / H]=[F e / H]+\log (0.638 f+0.362)} \\
f=10^{0.3}
\end{gathered}
$$

These equations solve for the absolute magnitude, $M_{i}$ and $M_{z}$, of an RR Lyrae using the period luminosity metallicity relations with the period, $P$, and 
metallicity, $Z$, of the star. $\log Z$ is derived from the metallicity ratio, $[M / H]$ found from the iron ratio to hydrogen, $[\mathrm{Fe} / \mathrm{H}]$, and the constant $f$.

The difference in brightness between the absolute magnitude $M$ and the calculated middle magnitude, $m$, is converted to distance in parsecs, $d$, similar to the inverse square law.

$$
\begin{gathered}
m-M=5 \log d-5 \\
d=10^{((m-M+5) / 5)}
\end{gathered}
$$

The star EZ Lyr from the Lyra constellation was selected for observation in this study. EZ Lyr is a visual double star in which one of the stars is an RR Lyre located at an RA and Dec of 281.9215, 35.99072 (Clementini et al., 2017). Its spectral type ranges from A9 to F6.5 and it has a metallicity estimated to be -1.56 (Suntzeff, Kraft, \& Kinman, 1994). Its period was found to be 0.525 days in 1959 (Preston, 1959). Photometric information about the field of EZ Lyr was available in the Panoramic Survey Telescope and Rapid Response System Pan-STARRS, (Chambers et al., 2016) and the APASS (Henden et al., 2016) catalogues allowing it to be analyzed closer to compare period-luminosity and distance.

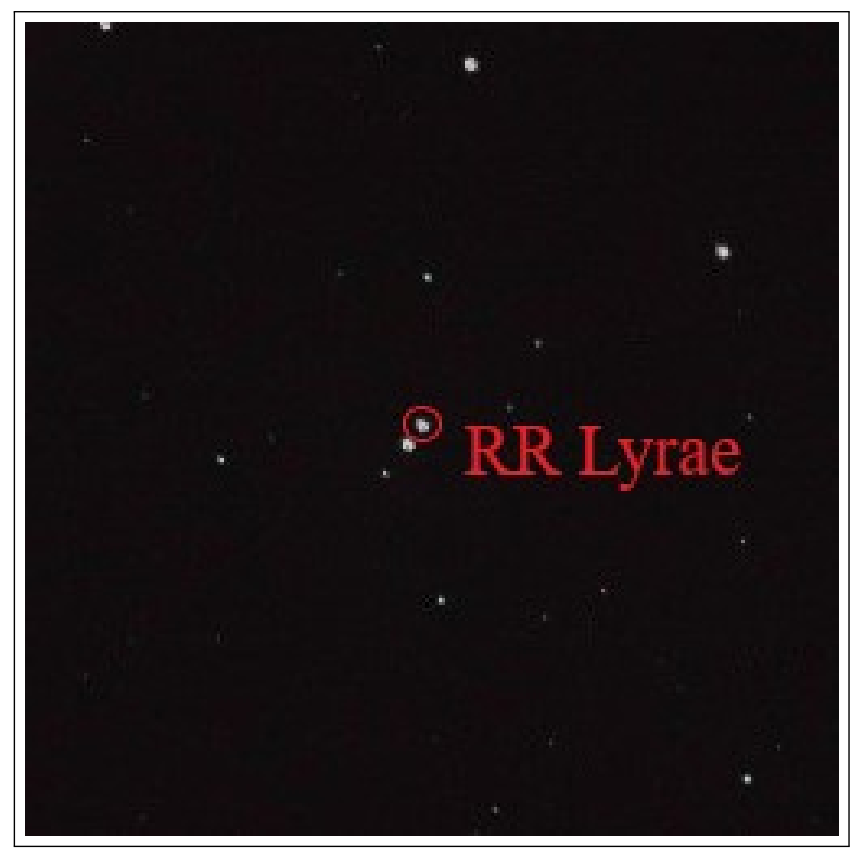

Fig. 1. Color image of EZ Lyr created with images taken with LCO using Fits Liberator and Gimp.

\section{OBSERVATIONS}

Images of EZ Lyr were taken robotically using the Las Cumbres Observatory (LCO) which is a global tele- scope network with twenty three telescopes located in seven sites around the world (Brown et al., 2013). This project specifically used the 0.4 meter SBIG telescopes over two different time periods. These observations were attempted in the $\mathrm{B}$-, $\mathrm{V}$-, i-, and z-bands every two hours during each cadence. In total, 63 successful observations were made in October 2019 and 30 in June 2020.

The final exposure times were 30 seconds for the Band z-bands and 10 seconds in V- and i-bands which produced light counts in the order of $10^{4}$ which was measured using AstroImageJ (Collins, Kielkopf, Stassun, \& Hessman, 2017). A misidentification error was made because of the close proximity to the other star in the EZ Lyr system causing initial light calculations to be double the true value for the RR Lyrae EZ Lyr. The exposure time for these images were originally half the appropriate value for the first approximately 20 images. These images were significantly noisier than the newer data, but due to time constraints, the clearer images from this set were still used for analysis.

Each image taken by LCO was then processed by the Our Solar Siblings Pipeline that automatically performed aperture and point spread function (PSF) photometry on each image (Fitzgerald, 2018). Since EZ Lyr is within a multiple star system, point source extractor (PSFex), a type of PSF photometry, produced the clearest data as it was able to isolate the luminosity of the variable star, therefore it was used for final calculations (Bertin, 2011).

\section{METHODS}

After the observations were completed, the data was processed through a Python code program called Astrosource which was provided by Our Solar Siblings (Fitzgerald, Gomez, Salimpour, Singleton, \& Wibowo, 2021). Each color filter was run individually through the five different scripts producing four sets of light curves. As the images were processed, the code first identified common stars and then chose stable comparison stars for the variable EZ Lyr whose luminosity was then calibrated using Pan-STARRS and APASS photometry data. Light curves and period calculations were then produced using two methods, string calibration and phase dispersion minimization (PDM)(Stellingwerf, 1978; Dworetsky, 1983; Altunin, Caputo, \& Tock, 2020).

Before being able to calculate distance, several values were found. The luminosity of EZ Lyr in each 
filter was estimated using the middle magnitude of the measured calibrated values. LogZ was determined using the $[\mathrm{Fe} / \mathrm{H}]$ of EZ Lyr (Cáceres \& Catelan, 2008). The period-luminosity-metallicity relationships were then used to convert the middle and absolute magnitude into distance measurements. To account for interstellar dust that scatters shorter wavelengths, observations in the B-filter were necessary to adjust measurements in other filters using interstellar reddening, $(\mathrm{E}(\mathrm{B}-\mathrm{V}))$. The value $(\mathrm{E}(\mathrm{B}-\mathrm{V}))$ was estimated by incriminating the number until the variance between the distance measurements in the V-, i-, and $\mathrm{z}$ - bands was minimized when accounting for extinction caused by $\mathrm{E}(\mathrm{B}-\mathrm{V})$. The following equation was used for this process where $A_{v}$ is extinction in the V-band which is used to modify the distance equation (Cardelli, Clayton, \& Mathis, 1989; Whitford, 1958).

$$
\begin{gathered}
A_{v}=3.1 E(B-V) \\
d=10^{\left(\left(m-M+5-A_{v}\right) / 5\right)}
\end{gathered}
$$

\section{RESULTS}

The period of EZ Lyr was estimated to be $0.5253 \pm$ 0.0007 days by averaging the periods found in the four filters, shown in Table 2, ignoring one significant outlier (PDM for B). The light curves displayed in Figure 2 show the characteristic "shark tooth" shape of an RRa type star.

\begin{tabular}{cccc}
\hline Filter & Mid Mag & Amplitude & Error \\
\hline B & 11.890 & 1.719 & 0.012 \\
V & 11.591 & 1.393 & 0.016 \\
ip & 11.388 & 1.009 & 0.022 \\
zs & 11.429 & 0.819 & 0.015 \\
\hline
\end{tabular}

Table 1. Luminosity Magnitude Measurements: The luminosity of each filter was calibrated from differential magnitude using data from PanSTARRS (Chambers et al., 2016).

$\log Z$ was calculated to be -3.11 after using the $[\mathrm{Fe} / \mathrm{H}]$ value of -1.56 in the following equations from Catelan.

$$
\begin{gathered}
\log Z=[M / H]-1.765 \\
{[M / H]=[F e / H]+\log (0.638 f+0.362)} \\
f=10^{0.3}
\end{gathered}
$$

Distance measurements were produced for V-, i-, and $\mathrm{z}$ - band filters, where the Viz value is the average of the three. The E(B-V) was adjusted to 0.08 at which the variance between the $\mathrm{V}$-, i-, and z-band distance measurements was 19.8 parsecs. The final calculation for the distance of EZ Lyr using the period luminosity relationship was $1406 \pm 32$ parsecs. Also listed Table 3 for comparison is the GAIA distance of $1523 \pm 60$ parsecs which was calculated using parallax.

\section{DISCUSSION}

The period of $0.5253 \pm 0.0007$ days is supported by previous measurements of 0.525 days and the V-band middle magnitude of 11.591 is comparable to other sources that report 11.3 and 11.58 (Preston, 1959; Høg et al., 2000). Possible causes of the error can be attributed to the initial calculation of exposure times that made the first set of data less exposed. This was the result of using a large aperture in AstroImageJ that encompassed both stars in the EZ Lyr system instead of isolating the RR Lyrae variable. The effect of this was minimized by collecting additional data several months later. This was mainly done as an attempt to collect data points in the sharp rise section of the light curve, but unfortunately, only a couple observations during this stage succeeded. This could have been caused by several factors such as the star's position in the sky being out of range of the telescopes or even bad visibility from weather. This gap does not seem to have impacted the final results as there was ample data in the other stages.

Other complications in data processing arose from the nature of EZ Lyr being a double star. It was at times hard to understand the celestial coordinate system and this led to questioning where to set the coordinates for analysis. In retrospect, it seems logical to focus on a single star, but there were attempts made to process the data while targeting the space between the stars and the two stars individually. As expected, only one of those positions produced coherent data, which was the RR Lyrae variable. Another confusion as a result was that it was briefly thought the variable star had been misclassified and was actually the other star in the system. This was cleared up after consulting Aladin and recognizing that the LCO images were flipped and it did in fact match previous findings (Bonnarel et al., 2000).

Applying the period-luminosity-metallicity mathematical relations was a simple process done in several steps on Excel using a prepared spreadsheet. The 
a)

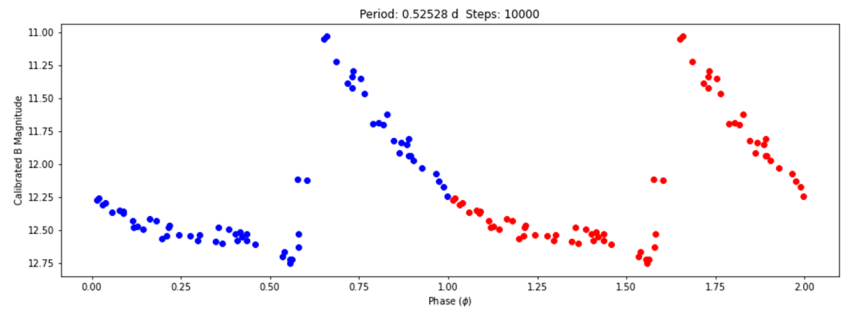

c)

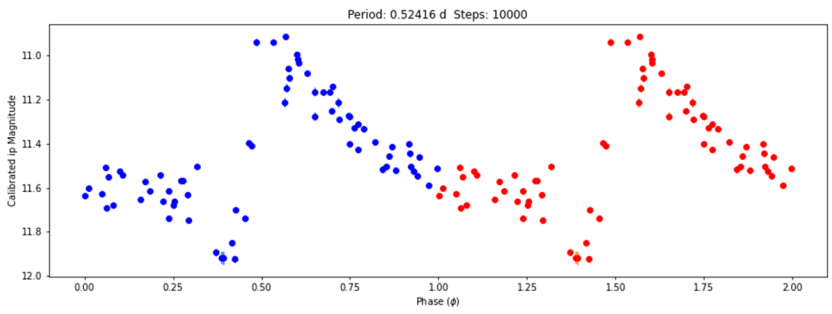

e)

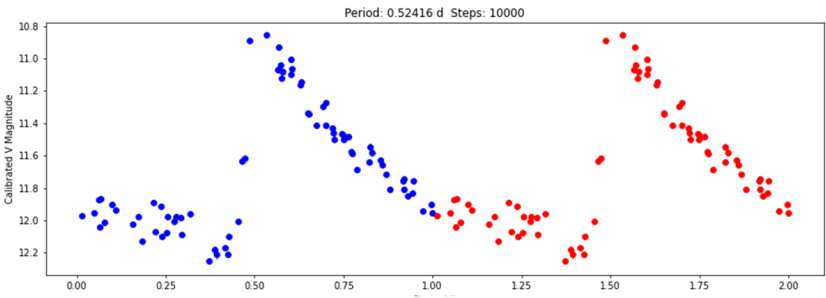

g)

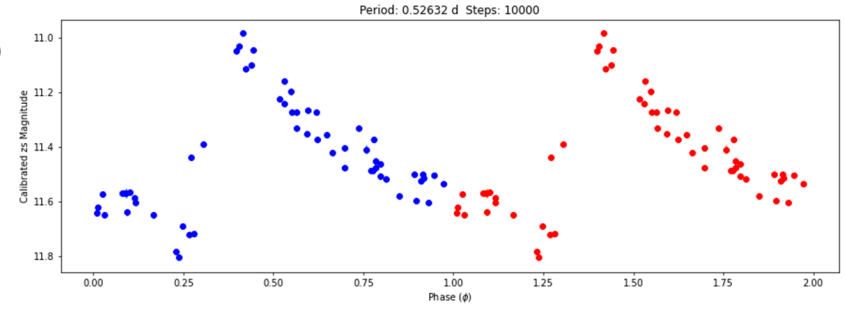

b)

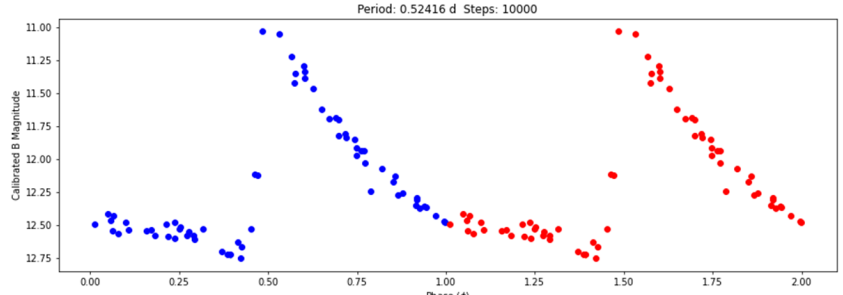

d)

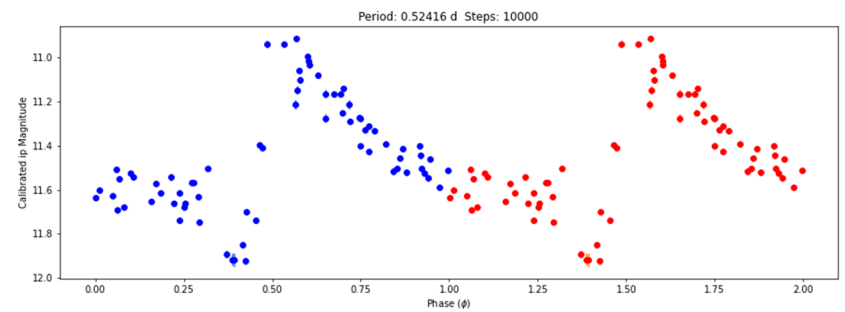

f)

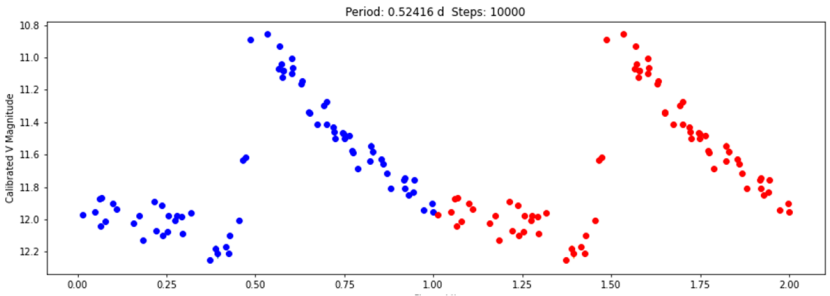

h)

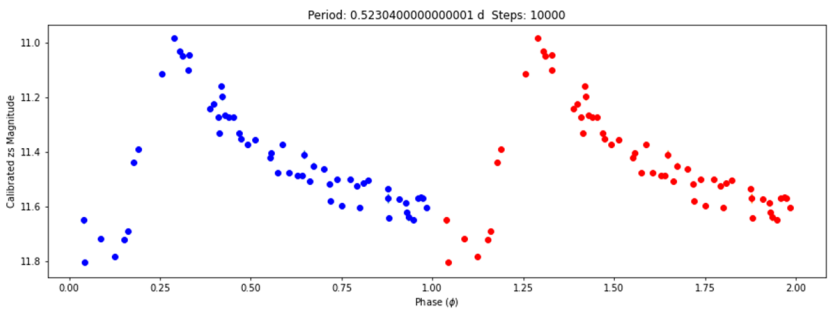

Fig. 2. Light curves for EZ Lyr in the B-, i-, V, and $z$ bands are shown above. The filters are as follows: B-band $(a, b)$, i-band (c, d), V-band (e, f), and z-band ( $g, h)$. String method is used in (a, c, e, g) and PDM method is used in $(b, d, f, h)$. Two full cycles are displayed to better visualize the shape of the curve. Calibrated luminosity magnitudes are shown in Table 1 . Period values and error are provided in Table 2.

\begin{tabular}{ccccc}
\hline Filter & String & String Error & PDM & PDM Error \\
\hline B & 0.52528 & 0.0003 & 0.52200 & 0.0002 \\
ip & 0.52528 & 0.0004 & 0.52528 & 0.0003 \\
V & 0.52416 & 0.0003 & 0.52632 & 0.0003 \\
zs & 0.52496 & 0.0103 & 0.52576 & 0.0110 \\
\hline
\end{tabular}

Table 2. Period Measurements: The period, in days, for each filter was measured using both String and PDM methods. The values were consistent with each other and had small error due to the amount of observations. 


\begin{tabular}{ccc}
\hline Filter & Distance $(\mathrm{pc})$ & Error $(\mathrm{pc})$ \\
\hline GAIA & 1523 & 60 \\
V & 1415 & 59 \\
i & 1383 & 51 \\
z & 1420 & 54 \\
Viz & 1406 & 32 \\
\hline
\end{tabular}

Table 3. Distance Measurements: The GAIA measurement using parallax is shown for comparison with values obtained using period luminosity for $\mathrm{V}, \mathrm{i}$, and $\mathrm{z}$ - band, where Viz is the average. Uncertainty in these measurements is from the period and calibration errors. Each value is visualised in Figure 3.

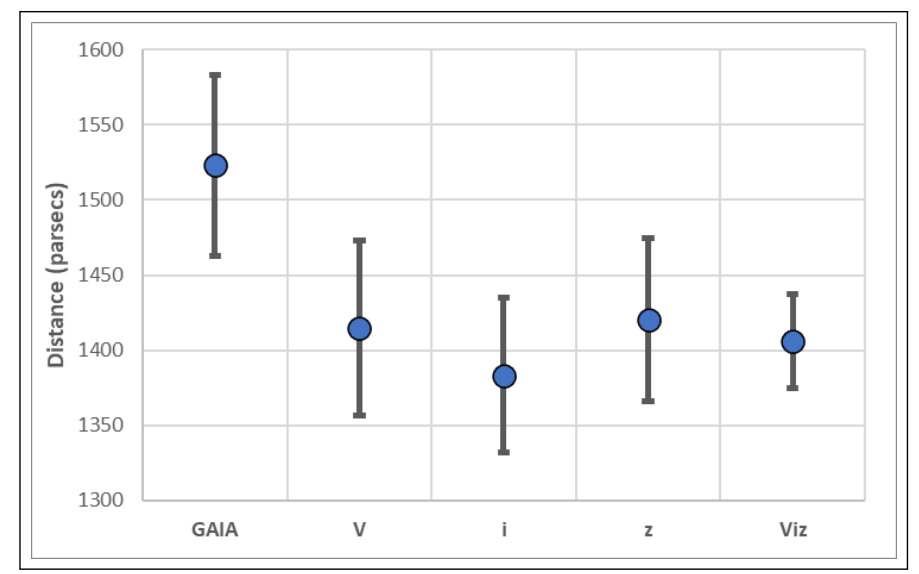

Fig. 3. Distance Measurements Graph: This is a plot of Table 3 showing how the distances in this study compare to GAIA. hardest part of this process was finding an appropriate interstellar reddening value $\mathrm{E}(\mathrm{B}-\mathrm{V})$. The number settled on 0.08 which is slightly lower than the value of $0.1293 \pm 0.0077$ from 2011 and $0.1503 \pm 0.0089$ from 1998 provided by the Schegel Dust Map Website (Schlegel, Finkbeiner, \& Davis, 1998). An E(B-V) $\approx$ 0.08 is a reasonable value given that the Schlegel dust maps give the total extinction along that line of sight and the star is in front of at least some of the dust. The image from the dust map site did not clearly separate the two stars and it was difficult to pinpoint exactly where the RR Lyrae should be because when entering the RA and Dec, the target on the map was shown in the gray area left of the bright stars marked in the picture. Because of this, the data from the website was used only as a rough estimate to begin refining the value until the variance was minimized.

Once the inputs were set, the final calculations were slightly shorter than GAIA's uncertainty margin. The calculations were precise after adjusting the interstellar reddening, therefore the accuracy of this value is a key factor in the legitimacy of the final data. Other measurements of interstellar reddening for EZ Lyr besides the Schegel Dust Map could not be found to support or oppose the value used here, so further evaluation of this value would be recommended.

\section{CONCLUSION}

This project showed that a reliable distance could be produced using a period luminosity metallicity relationship. The results for EZ Lyr were all within the same range of each other with reasonable uncertainty and are close to the GAIA distance which used parallax. In the future, when combined with results from other groups performing the same experiment on different RR Lyrae stars, there may prove to be substantial evidence that Catelan's method is as accurate as parallax distance measurements. Since this method does not rely on parallax angles, it could be used on more distant stars. A limitation of this method is that RR Lyraes tend to be fainter stars, however with longer exposure times and larger telescopes, it could still be possible to estimate distances to the more far off stars.

\section{FUNDING}

Student participation in this project was funded by the National Aeronautics and Space Administration under Grant No. NNX15AR73H issued through the Tennessee Space Grant Consortium. 

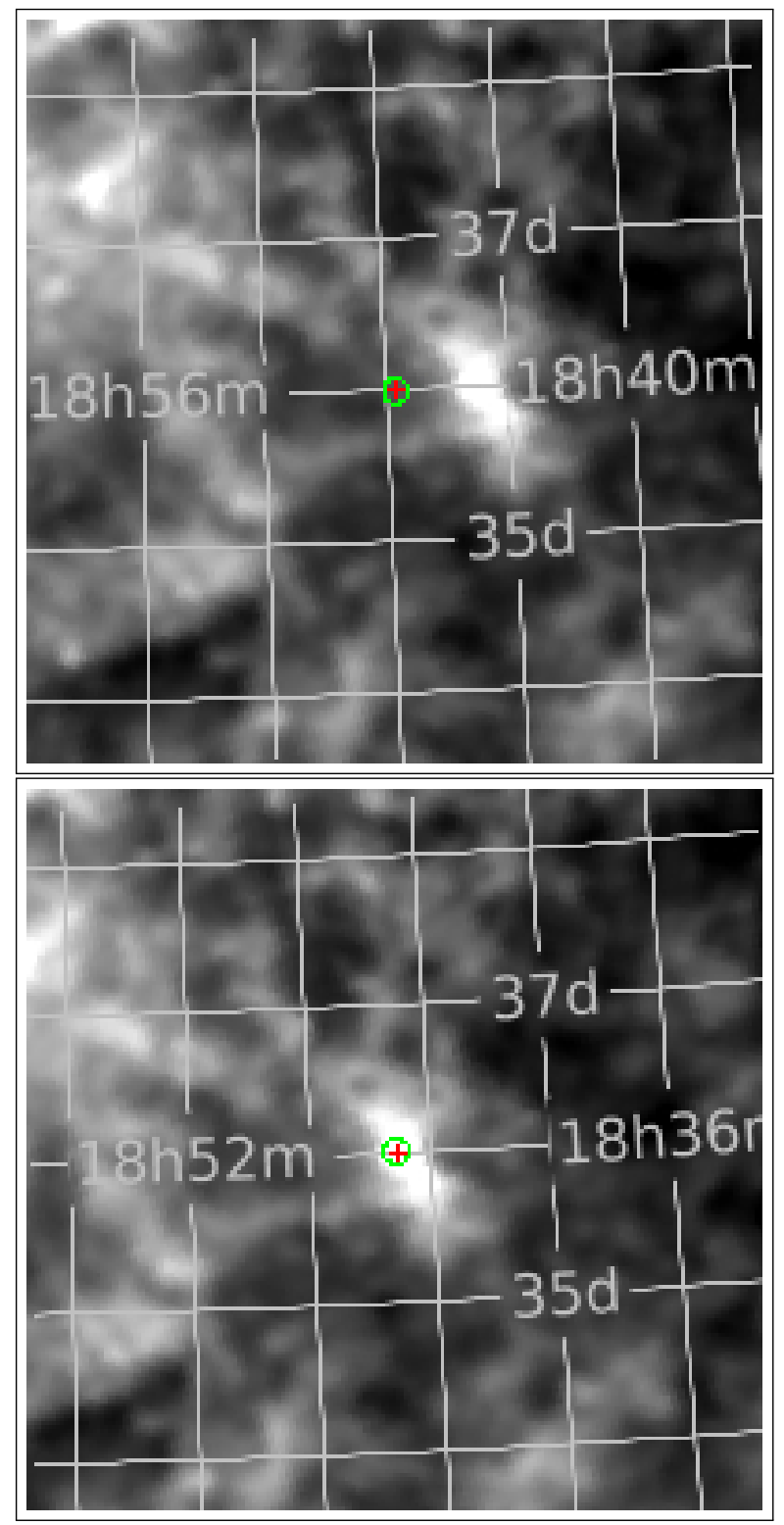

Fig. 4. Schegel Dust Map Targets: The first image shows the target automatically placed given the location of EZ Lyr. The second image is where the target was manually placed to better represent the actual location of EZ Lyr based off the bright points.

\section{ACKNOWLEDGMENTS}

This project was part of a research course run by Dr. Michael Fitzgerald of Our Solar Siblings who supported students throughout the course. Our Solar Siblings (OSS) is a inquiry based project to high school and undergraduate students interested in doing investigative research in astronomy. OSS provided access to their pipeline, educational materials, and time with the Las Cumbres Observatory.

Appreciation is also given to free programs utilized in this course such as Stellarium (https://stellarium.org/), Aladin (https://aladin.u-strasbg.fr/) , AstroImageJ (https://www.astro.louisville.edu/software/ astroimagej/), Gimp (https://www.gimp.org/), and Fits Liberator (https://www.spacetelescope .org/projects/fits_liberator/).

\section{REFERENCES}

Altunin, I., Caputo, R., \& Tock, K. (2020). Period of eclipsing binary epic 201458798. Astronomy Theory, Observations \& Methods, 1(1).

Bertin, E. (2011). Automated morphometry with sextractor and psfex. In Astronomical data analysis software and systems $x x$ (Vol. 442, p. 435).

Bonnarel, F., Fernique, P., Bienaymé, O., Egret, D., Genova, F., Louys, M., ... Bartlett, J. G. (2000). The aladin interactive sky atlas-a reference tool for identification of astronomical sources. Astronomy and Astrophysics Supplement Series, 143(1), 33-40.

Brown, T., Baliber, N., Bianco, F., Bowman, M., Burleson, B., Conway, P., ... others (2013). Las cumbres observatory global telescope network. Publications of the Astronomical Society of the Pacific, 125(931), 1031.

Cáceres, C., \& Catelan, M. (2008). The periodluminosity relation of rr lyrae stars in the sdss photometric system. The Astrophysical Journal Supplement Series, 179(1), 242.

Cardelli, J. A., Clayton, G. C., \& Mathis, J. S. (1989). The relationship between infrared, optical, and ultraviolet extinction. The Astrophysical Journal, 345, 245-256.

Chambers, K. C., Magnier, E., Metcalfe, N., Flewelling, H., Huber, M., Waters, C., ... others (2016). The pan-starrs1 surveys. arXiv preprint arXiv:1612.05560.

Clementini, G., Eyer, L., Ripepi, V., Marconi, M., Muraveva, T., Garofalo, A., ... others (2017). 
Gaia data release 1-testing parallaxes with local cepheids and rr lyrae stars. Astronomy \& Astrophysics, 605, A79.

Collins, K. A., Kielkopf, J. F., Stassun, K. G., \& Hessman, F. V. (2017). Astroimagej: Image processing and photometric extraction for ultra-precise astronomical light curves. The Astronomical Journal, 153(2), 77.

Dambis, A., Berdnikov, L., Kniazev, A. Y., Kravtsov, V., Rastorguev, A., Sefako, R., \& Vozyakova, O. (2013). Rr lyrae variables: visual and infrared luminosities, intrinsic colours and kinematics. Monthly Notices of the Royal Astronomical Society, 435(4), 3206-3220.

Dworetsky, M. (1983). A period-finding method for sparse randomly spaced observations or "how long is a piece of string?". Monthly Notices of the Royal Astronomical Society, 203(4), 917-924.

Fitzgerald, M. T. (2018). The our solar siblings pipeline: Tackling the data issues of the scaling problem for robotic telescope based astronomy education projects.

Fitzgerald, M. T., Criss, J., Lukaszewicz, T., Frew, D. J., Catelan, M., Woodward, S., ... McKinnon, D. H. (2012). Rr lyrae stars in the globular cluster ngc 6101. Publications of the Astronomical Society of Australia, 29(1), 72-77.

Fitzgerald, M. T., Gomez, E., Salimpour, S., Singleton, J., \& Wibowo, R. W. (2021). "astrosource": automating optical astronomy measurement, calibration and analysis for variable stellar sources from provided photometry. Journal of Open Source Software, 6(58), 2641.

Henden, A. A., Templeton, M., Terrell, D., Smith, T. C., Levine, S., \& Welch, D. (2016, January). VizieR Online Data Catalog: AAVSO Photometric All Sky Survey (APASS) DR9 (Henden+, 2016). VizieR Online Data Catalog, II/336.

Høg, E., Fabricius, C., Makarov, V. V., Urban, S., Corbin, T., Wycoff, G., ... Wicenec, A. (2000, March). The Tycho-2 catalogue of the 2.5 million brightest stars. , 355, L27-L30.

Preston, G. W. (1959). A spectroscopic study of the rr lyrae stars. The Astrophysical Journal, 130, 507.

Schlegel, D., Finkbeiner, D., \& Davis, M. (1998). Application of sfd dust maps to galaxy counts and $\mathrm{cmb}$ experiments. arXiv preprint astroph/9809230.

Stellingwerf, R. F. (1978). Period determination using phase dispersion minimization. The Astrophysical Journal, 224, 953-960.

Suntzeff, N. B., Kraft, R. P., \& Kinman, T. (1994). Summary of delta s metallicity measurements for bright rr lyrae variables observed at lick observatory and kpno between 1972 and 1987. The Astrophysical Journal Supplement Series, 93, 271-285.

Whitford, A. (1958). The law of interstellar reddening. The Astronomical Journal, 63, 201-207. 